\title{
Photometric study for near-Earth asteroid (155140) 2005 UD
}

\author{
Huang, J.-N., ${ }^{\mathrm{a}, \mathrm{b}, \mathrm{c}}$, Muinonen, K. ${ }^{\mathrm{d}, \mathrm{e}}$, Chen, T. ${ }^{\mathrm{f}}$, Wang, X.-B. ${ }^{\mathrm{a}, \mathrm{b}, \mathrm{c}, *}$ \\ ${ }^{a}$ Yunnan Observatories, Chinese Academy of Sciences, Kunming, 650216, China \\ ${ }^{b}$ School of Astronomy and Space Sciences, University of Chinese Academy of Sciences, \\ Beijing, 100049, China \\ ${ }^{c}$ Key Laboratory for Structure and Evolution of Celestial Objects, CAS, Kunming, \\ 650216, China \\ ${ }^{d}$ Department of Physics, University of Helsinki, Gustaf Hällströmin katu 2, P.O. Box 64 , \\ FI-00014 U. Helsinki, Finland \\ ${ }^{e}$ Finnish Geospatial Research Institute, Geodeetinrinne 2, FI-02430 Masala, Finland \\ ${ }^{f}$ Corona borealis Observatiories, Ali, China
}

\begin{abstract}
The Apollo-type near-Earth asteroid (155140) 2005 UD is thought to be a member of the Phaethon-Geminid meteor stream Complex (PGC). Its basic physical parameters are important for unveiling its origin and its relationship to the other PGC members as well as to the Geminid stream. Adopting the Lommel-Seeliger ellipsoid method and $H, G_{1}, G_{2}$ phase function, we carry out spin, shape, and phase curve inversion using the photometric data of 2005 UD. The data consists of 11 new lightcurves, 3 lightcurves downloaded from the Minor Planet Center, and 166 sparse data points downloaded from the Zwicky Transient Facility database. As a result, we derive the pole solution of $\left(285^{\circ} .8_{-5.3}^{+1.1},-25^{\circ} .8_{-12.5}^{+5.3}\right)$ in the ecliptic frame of J2000.0 with the
\end{abstract}

\footnotetext{
${ }^{*}$ Corresponding author: Xiaobin Wang wangxb@ynao.ac.cn, huangjianing@ynao.ac.cn
} 
rotational period of $5.2340 \mathrm{~h}$. The corresponding triaxial shape (semiaxes $a>b>c)$ is estimated as $b / a=0.76_{-0.01}^{+0.01}$ and $c / a=0.40_{-0.01}^{+0.03}$. Using the calibrated photometric data of $2005 \mathrm{UD}$, the $H, G_{1}, G_{2}$ parameters are estimated as $17.19_{-0.09}^{+0.10} \mathrm{mag}, 0.573_{-0.069}^{+0.088}$, and $0.004_{-0.021}^{+0.020}$, respectively. Correspondingly, the phase integral $q$, photometric phase coefficient $k$, and the enhancement factor $\zeta$ are $0.2447,-1.9011$, and 0.7344 . From the values of $G_{1}$ and $G_{2}, 2005$ UD is likely to be a C-type asteroid. We estimate the equivalent diameter of 2005 UD from the new $H$-value: it is $1.30 \mathrm{~km}$ using the new geometric albedo of 0.14 .

Keywords: Near-Earth-Asteroid, Photometry, Shape inversion, Phase function

\section{Introduction}

Near-Earth asteroids (NEAs) derive from the main belt of asteroids and are composed of planetesimal material remaining from the early stages of the Solar System. They contain important information about the Solar System's formation and evolution. Thus, the physical study for NEAs can allow us to know more about the history of the Solar System, including that of the asteroids. Asteroid 2005 UD has been discovered by the Catalina Sky Survey in 22 October 2005, and, according to its orbit, it has been classified as an Apollo-type NEA. Together with (3200) Phaethon and 1996 YC, 2005 UD belongs to the Phaethon-Geminid stream Complex, briefly PGC.Ohtsuka et al. (2006) investigated the orbital evolution of 2005 UD and Phaethon, and suggested the object may be a split nucleus of Phaethon. As a member 
of PGC, the physical properties of 2005 UD should provide information about its origin and relationship to the other PGC members and to the Geminid meteors.

Several groups have carried out physical studies of 2005 UD. Ohtsuka et al. (2006) inferred that 2005 UD is a km-size object. Jewitt and Hsieh (2006)'s data of 2005 UD showed a periodic brightness variation of $5.2492 \mathrm{~h}$ with an amplitude of $0.4 \mathrm{mag}$. They also estimated the diameter of 2005 UD to be $1.3 \pm 0.1 \mathrm{~km}$, assuming a geometric albedo of 0.11 (the geometric albedo of Phaethon). Kinoshita et al. (2007) determined the rotation period for $2005 \mathrm{UD}$, and found variation of color indices with rotational phase. They derived a rotation period of $5.23 \mathrm{~h}$ with a lightcurve amplitude of $0.44 \pm 0.02 \mathrm{mag}$. Their multi-band photometric observations suggested that 2005 UD is of F or B type. Later, Kasuga and Jewitt (2008) suggested that 2005 UD is a C-type asteroid, based on the color index. Kasuga and Jewitt (2008) determined the absolute magnitude of 2005 UD to be $17.23 \pm 0.03 \mathrm{mag}$, and derived a diameter of $1.2 \pm 0.1 \mathrm{~km}$, assuming an albedo of $0.11 \pm 0.02$. Jewitt (2013) re-determined the surface color indices of 2005 UD and estimated its absolute magnitude to be $17.08 \mathrm{mag}$, assuming the $H, G$ phase function slope of $G=0.15$. Based on the WISE observations, Masiero et al. (2019) determined the geometric albedo of 2005 UD to be $0.14 \pm 0.09$, and derived a diameter of $1.2 \pm 0.4 \mathrm{~km}$. Recently, Krugly et al. (2019) and Warner and Stephens (2019) reported a similar rotation period for 2005 UD. Krugly et al. (2019) determined the linear phase-angle coefficient of $0.043 \mathrm{mag} \mathrm{deg}^{-1}$. As for the spin and shape parameters, there is no further information preceding 
our study.

For understanding the physical properties of 2005 UD, we have carried out 11 nights of photometric observations in 2018. In addition, we download the photometric data of 2005 UD from the Zwicky Transient Facility (ZTF) and Minor Planet Center (MPC) databases. ZTF is a robotic time-domain astronomical sky survey. The small Solar System bodies are important targets of the survey.In total, 166 data points of 2005 UD are downloaded from the ZTF database.

In the article, we present our new photometric observations of 2005 UD and our results of the photometric analyses of 2005 UD with the inverse methods based on Lommel-Seeliger ellipsoids (Muinonen et al. (2015)) and $H, G_{1}, G_{2}$ phase curves (Muinonen et al. (2010)). Therefore, Sect. 2 shows the observations and data reductions for the object, Sect. 3 introduces the methods of lightcurve inversion and phase curve inversion, together with the results and discussion. In the last section, a summary is presented.

\section{Observations and data reductions}

We obtained 11 nights of photometric observations of 2005 UD in October 2018 using the $30-\mathrm{cm}$ telescope and a $3326 \times 2504$ CCD at the Corona borealis observatory (code N55). The field of view (FOV) of the CCD is $28^{\prime} .4 \times 21^{\prime} .4$. The data were gathered through $\mathrm{C}$ (Clear), $\mathrm{R}$, or $\mathrm{V}$ filters depending on the weather conditions and the signal-to-noise ratio. During the observations, the weather conditions were acceptable with good seeing, and the status of the instrument was fine. Additional observational information is shown in 
Table 1. During the photometric observations, sidereal tracking and short exposure times were utilized. During the observations, the sky-plane motion of 2005 UD was large at about $8^{\prime \prime} .7 \sim 3^{\prime \prime} .5 / \mathrm{min}$. Consequently, the telescope pointing was shifted once or twice in some nights to keep the object in the FOV.

The photometric images were reduced according to the standard procedures with the IRAF software. The effects of bias, flat field, and dark current were corrected first. The cosmic rays in the images were removed properly. The magnitudes of the celestial objects in the scientific frames were measured using the Apphot task of IRAF with an optimum aperture. We tried 3-5 apertures ranging 2.0-2.3 times the full width at half maximum (FWHM) to find the optimum aperture giving the minimum dispersion of the lightcurve points.

Some systematic errors in the photometric data, related to atmospheric extinction and temporal or positional changes of stars in the CCD, were simulated with the aid of reference stars in the images using the coarse decorrelation method (Collier Cameron et al. (2006). Wang et al. (2013)) and the SYSREM method (Tamuz et al. (2005)). The former performs a coarse initial de-correlation by referencing each star's magnitude to its own mean, finding small night-to-night and frame-to-frame differences in the zero point. After the coarse de-correlation, the reduced magnitudes of celestial objects can be derived (see Eq. 1d below) by removing the biases due to the objects' mean magnitude in each night and zero-point in each frame. The latter method simulates the low-level systematic errors in the reduced magnitudes 
by those chosen reference stars. Then those simulated low-level errors, or, say, patterns in the reduced magnitudes are removed from the reduced magnitudes of the 2005 UD. The ratio of signal to noise of the lightcurves (reduced magnitudes in one night) is therefore enhanced. The time stamp of each observation of the asteroid is corrected for the light travel time. The distance effects on the asteroid's magnitude are also corrected by the formula $-5 \log (r \Delta)$.In total, 2206 data points were obtained in 11 nights. For the aim of shape inversion, the relative intensities of 2005 UD are used which are derived by normalizing the mean intensity of each lightcurve to unity.

For the phase curve analysis of 2005 UD, photometric data obtained in different nights and/or with different filters need to be converted into the same photometric system, e.g., the standard V-band magnitudes. In this work, we firstly transformed the instrumental magnitudes of celestial objects into the $r^{\prime}$ band of the Carlsberg Meridian Catalogue 15 (CMC15). The relationship between the instrumental magnitude and the $r^{\prime}$-band of CMC15 (Eq. 2) is derived by using stable reference stars in the images. The reference stars are chosen based on a threshold in the intrinsic variance, e.g., 0.01 mag in the case of 2005 UD's observations. The intrinsic variance of the stars $\sigma_{s(i)}$ in a night and the variance of the observations $\sigma_{t(j)}$ (the variance of reference stars' magnitudes in a image after removing their means), are output by the coarse de-correlation method (for details, see Collier Cameron et al. (2006)). Briefly, the two variances above are estimated iteratively by minimizing the value of $\chi^{2}$ (Eq. 1a below), providing zero points of the stars' magnitudes $\hat{M}_{i}$ in a night and the zero point of each frame $\hat{Z}_{j}$ (calculated with Eqs. $1 \mathrm{~b}$ and 

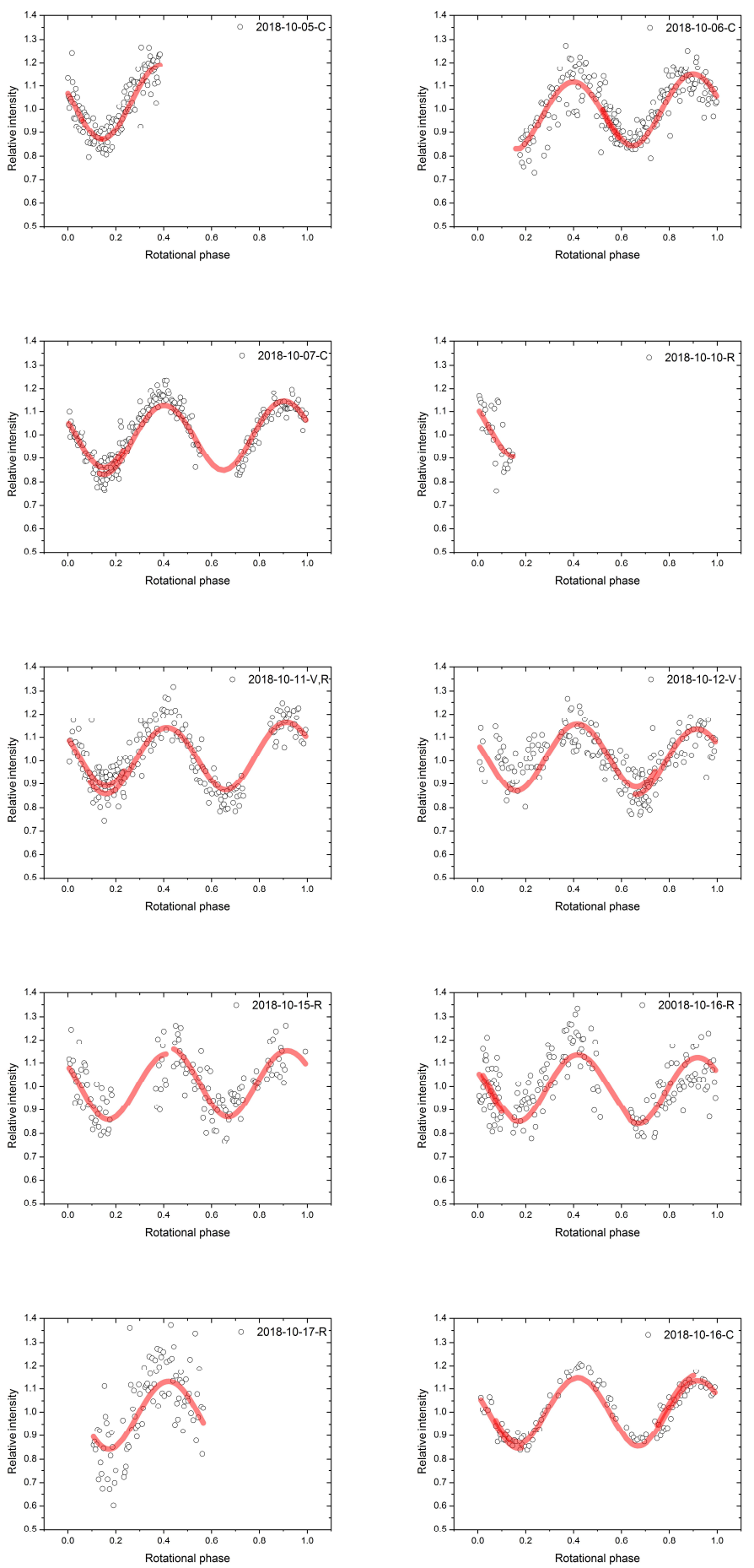

Figure 1: Ten lightcurves of 2005 UD folded with the period of $5.2340 \mathrm{~h}$. Red lines are best-fit model values. 
Table 1: Information on the phototometric observations of near-Earth asteroid 2005 UD.

\begin{tabular}{|c|c|c|c|c|c|c|c|}
\hline $\begin{array}{l}\text { Date } \\
\text { (UT ) }\end{array}$ & $\begin{array}{c}r \\
(\mathrm{au})\end{array}$ & $\begin{array}{c}\Delta \\
(\mathrm{au})\end{array}$ & $\begin{array}{c}\alpha \\
(\operatorname{deg})\end{array}$ & Mag-V & Filter & $\mathbf{N}$ & Data source \\
\hline $2018 / 10 / 05$ & 1.219 & 0.245 & 21.301 & 15.9 & $C$ & 135 & Ourdata \\
\hline $2018 / 10 / 06$ & 1.232 & 0.252 & 17.588 & 15.9 & $C$ & 229 & Ourdata \\
\hline $2018 / 10 / 07$ & 1.246 & 0.261 & 14.198 & 15.9 & $C$ & 263 & Ourdata \\
\hline $2018 / 10 / 10$ & 1.286 & 0291 & 5.671 & 15.8 & $R$ & 36 & Ourdata \\
\hline $2018 / 10 / 11$ & 1.299 & 0.302 & 3.152 & 15.8 & $V, R$ & 227 & Ourdata \\
\hline 2018/10/12 & 1.312 & 0.315 & 1.790 & 15.7 & $C$ & 52 & $R$, Stephens \\
\hline 2018/10/12 & 1.312 & 0.315 & 0.964 & 15.7 & $V$ & 204 & Ourdata \\
\hline 2018/10/13 & 1.325 & 0.327 & 1.726 & 15.7 & $V$ & 290 & Ourdata \\
\hline 2018/10/14 & 1.338 & 0.341 & 3.685 & 15.8 & $V$ & 292 & Ourdata \\
\hline $2018 / 10 / 15$ & 1.350 & 0.354 & 4.642 & 16.0 & $C$ & 132 & $R$, Stephens \\
\hline $2018 / 10 / 15$ & 1.350 & 0.354 & 5.414 & 16.1 & $R$ & 141 & Ourdata \\
\hline $2018 / 10 / 16$ & 1.363 & 0.369 & 6.455 & 16.3 & $C$ & 116 & $R$, Stephens \\
\hline $2018 / 10 / 16$ & 1.363 & 0.369 & 7.130 & 16.5 & $R$ & 185 & Ourdata \\
\hline $2018 / 10 / 17$ & 1.375 & 0.383 & 8.827 & 16.6 & $R$ & 208 & Ourdata \\
\hline $2017 / 10 / 27-2019 / 7 / 20$ & & & & & & 166 & $Z T F^{\text {Facility }}$ \\
\hline
\end{tabular}

Note that $r$ and $\Delta$ are heliocentric and topocentric distances of the asteroid, $\alpha$ is the solar phase angle, Mag- $\mathrm{V}$ is the mean of the observed $\mathrm{V}$-band magnitudes in a night, $\mathrm{N}$ is the number of data points, and $Z T F^{\text {Facility }}$ refers to IRSA, Spitzer, WISE, Herschel, Planck, SOFIA, IRTF, IRAS, and MSX.

1c). The quantities $\hat{M}_{i}$ and $\hat{Z}_{j}$ are re-computed when we have new values for $\sigma_{s(i)}$ and $\sigma_{t(j)}$. The iterative procedure is ended when the four quantities above no longer change significantly. For a given reference star $i$, the errors in the reduced magnitudes $r_{i, j}$ in a night, $j$ denoting the index of a frame, are finally random, essentially realizations of white noise. The following equation 
gives a mathematical description of the procedures outlined above:

$$
\begin{aligned}
& \chi^{2}=\sum \frac{\left(m_{i j}-\hat{M}_{i}-\hat{Z}_{j}\right)^{2}}{\sigma_{i j}^{2}+\sigma_{s(j)}^{2}+\sigma_{t(i)}^{2}}, \\
& \hat{M}_{i}=\frac{\sum_{i}\left(m_{i j}-\hat{Z}_{j}\right) * w_{i j}}{w_{i j}}, \quad w_{i j}=\frac{1}{\sigma_{i j}^{2}+\sigma_{t(j)}^{2}}, \\
& \hat{Z}_{j}=\frac{\sum_{i}\left(m_{i j}-\hat{M}_{i}\right) * u_{i j}}{u_{i j}}, \quad u_{i j}=\frac{1}{\sigma_{i j}^{2}+\sigma_{s(i)}^{2}}, \\
& r_{i j}=m_{i j}-\hat{M}_{j}-\hat{Z}_{i} .
\end{aligned}
$$

Here $m_{i j}$ represents the observed magnitude of star $i$ in frame $j$, and $\sigma_{i j}$ is the corresponding observational uncertainty.

The magnitude zero points of selected reference stars in a night $\hat{M}_{i}$ are applied to fit the relationship of Eq. 2:

$$
\hat{M}_{i}(C / R / V)=M_{(C / R / V) 0}+M_{\left(r^{\prime}\right) i}+k_{(C / R / V)} \times\left(J_{i}-K s_{i}\right)
$$

In detail, the parameters $M_{(C / R / V) 0}$ and the color index coefficient $k_{(C / R / V)}$ in Eq. 2 are fitted by comparing the $\hat{M}_{i}$ values of selected stars to their values $M_{\left(r^{\prime}\right)} i$ in CMC15. The color indices $\left(J_{i}-K s_{i}\right)$ of reference stars come from the 2MASS catalogue. The $r^{\prime}$-band of $\mathrm{CMC} 15$ is the same as that in the Sloan Digital Sky Survey. In Eq. 2, $i$ denotes the reference star and C, R, and $\mathrm{V}$ denote different filters. Using the derived parameters $M_{(C / R / V) 0}$ and $k_{(C / R / V)}$, the mean magnitude of the asteroid in a given night is transformed into the $r^{\prime}$-band of the CMC15 photometric system, denoted by $M\left(r^{\prime}\right)_{a s t}$. For no measurement value for 2005 UD's color index $(J-K s)_{a s t}$, we temporally use that of Phaethon's at 0.275 * . Then the $r^{\prime}$-band magnitudes of the

\footnotetext{
${ }^{*}$ https://sbnapps.psi.edu/ferret/SimpleSearch/results.action
} 
asteroid are converted into the standard V-band magnitude by another linear relationship (Dymock and Miles (2009))

$$
V_{a s t}=0.6278 \times(J-K s)_{a s t}+0.9947 \times M\left(r^{\prime}\right)_{a s t} .
$$

The calibrated mean magnitude $V_{\text {ast }}$ of each lightcurve of 2005 UD are used in the phase curve inversion: the results are shown in Fig. 2. The individual data points of the asteroid in a night are calibrated by adding the calibrated mean magnitude $V_{a s t}$ into the reduced magnitudes in that night.

In addition, we have downloaded, from the MPC Asteroid Lightcurve Photometry Database $\mathrm{f}^{\dagger}$, three dense lightcurves of 2005 UD observed on 12, 15, and 16 October 2018. These data have been observed through a clear filter and have been converted into the V-band. We corrected the distance effects on the magnitude and light travel time in each recorded time stamp.

Finally, we have downloaded sparse photometric data of 2005 UD from


the Samuel Oschin 48-inch Schmidt telescope with a new Mosaic CCD of a 47 square-degree FOV. This observational system is equipped with three filters: ZTF-g, ZTF-r, and ZTF-i. The limiting magnitude in the ZTF-r band is 20.7 mag with a $5-\sigma$ detection threshold in a 30-s exposure. It can scan more than $3750 \mathrm{deg}^{2}$ per hour. The ZTF data reduction follows the data processing system of the Palomar Transient Factory (PTF) survey. They use a fixed aperture of 8 pixels to obtain instrumental magnitudes and carry out

\footnotetext{
${ }^{\dagger}$ http://alcdef.org/PHP/alcdef_GenerateALCDEFPage.php

${ }^{\ddagger}$ https://irsa.ipac.caltech.edu/applications/ztf/
} 
photometric calibration with the reference stars in the SDSS catalogue and in the ZTF images (Ofek et al. (2012), Laher et al. (2014), Bellm et al. (2019)). The downloaded 166 photometric data points of 2005 UD span from 2017 October to 2019 July. Those data points have been converted into the mean standard V band (Ofek et al. (2012). Bellm et al. (2019)). For those data, we have corrected the distance effects on the magnitude and light travel time in the recorded time stamps (see Fig. 2 ; the black circles denote the ZTF data).

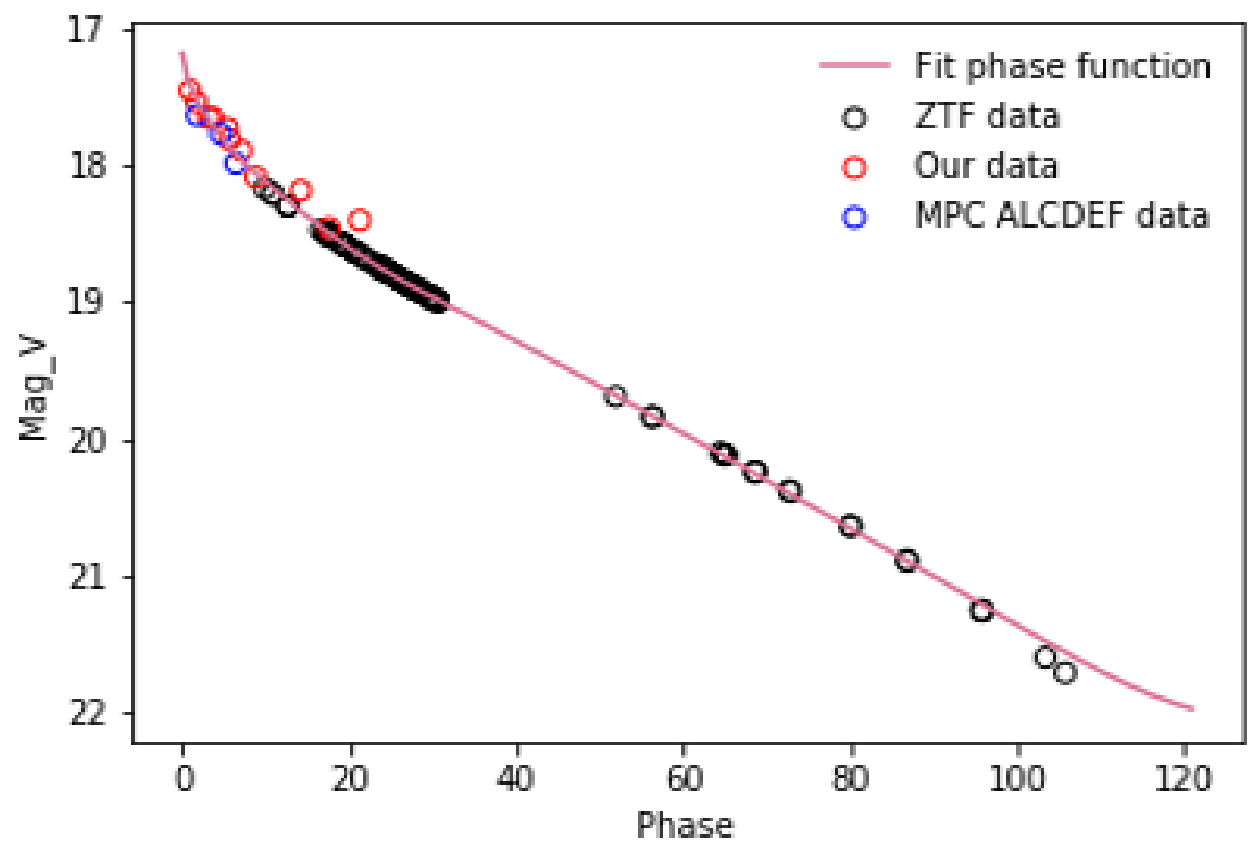

Figure 2: Phase curve of 2005 UD. 


\section{Photometric analysis}

\subsection{Lommel-Seeliger elliposid model}

An asteroid's brightness results from the scattering of sunlight by its surface. How bright the asteroid truly is depends on its size, shape, orientation, and surface scattering properties. The surface scattering properties determine the asteroid's geometric albedo. The brightness is standardized by locating, fictitiously, the asteroid at a 1-au distance from the Sun as well as from the observer. As for the effects on magnitude due to the varying distance of asteroid from the Sun and the observer, we account for them by the formula $-5 \log (r \Delta)$. The brightness of the asteroid varies with the solar phase angle, the angle between the Sun and the observer as seen from the object. Such brightness variation is called the phase curve. Most asteroids have an irregular shape. For a spinning nonspherical asteroid, the brightness varies as a function of rotational phase due to the varying illuminated and visible parts of surface. The resulting brightness curve is called the lightcurve. The shape of the lightcurve of an asteroid is usually dominated by the shape of the asteroid. The lightcurve shape varies from one apparition to another due to the changes in the so-called aspect angles, the angles between the spin pole direction and the line of sight or the direction of sunlight. The lightcurve of an asteroid contains information on the asteroid's physical properties, that is, size, albedo, spin status, and shape, and it depends on the observation geometry. We may infer the asteroid's properties from the lightcurves if the lightcurves span a sufficiently large selection of observa- 
tional geometries. The procedure is called lightcurve inversion, in which a brightness model is established to invert the asteroid's properties assuming a shape model and a surface scattering law. Normally, scattering laws such as the Lommel-Seeliger law, Lambert law, Hapke model (Hapke (2012)) and Lumme-Bowell model (Bowell and Lumme (1979)) are used. As for the shape model, a triaxial ellipsoid model or a more complex convex shape model can be introduced, depending on the characteristics of the lightcurves.

The regular shape of lightcurves of 2005 UD (Fig. 1) with two peaks implies a regular shape of the asteroid. That is the main reason why we use, in the lightcurve inversion, the triaxial ellipsoid model with the Lommel-Seeliger scattering law (LS ellipsoid; Muinonen et al. (2015)). For an elementary facet $d A$ on the asteroid's surface, the brightness of the facet with the LommelSeeliger law can be written as

$$
d L=\frac{1}{4} F_{0} \mu_{0} \varpi_{0} P(\alpha) \frac{1}{\mu+\mu_{0}} \mu d A
$$

where $\pi F_{0}$ is the incident solar flux density on the facet, $\varpi_{0}$ is the singlescattering albedo, $\alpha$ is the solar phase angle, and $P(\alpha)$ is the single-scattering phase function.

The brightness integrated over an ellipsoid surface with the LommelSeeliger scattering law is given as

$$
\begin{aligned}
& L\left(\alpha, e_{\odot}, e_{\oplus}\right)= \frac{1}{8} \\
& \pi F_{0} a b c \varpi_{0} P(\alpha) \frac{S_{\odot} S_{\oplus}}{S}\left\{\cos \left(\lambda^{\prime}-\alpha^{\prime}\right)+\cos \lambda^{\prime}+\sin \lambda^{\prime} \sin \left(\lambda^{\prime}-\alpha^{\prime}\right)\right. \\
&\left.\times \ln \left[\cot \frac{1}{2} \lambda^{\prime} \cot \frac{1}{2}\left(\alpha^{\prime}-\lambda^{\prime}\right)\right]\right\}
\end{aligned}
$$

Here, $e_{\odot}$ and $e_{\oplus}$ denote the unit vectors of solar and viewer directions, $a, b$, 
and $c$ are the three semimajor axes of the ellipsoid, and the auxiliary quantities $S_{\odot}, S_{\oplus}, \alpha^{\prime}, \lambda^{\prime}$ are functions of the illumination and viewing geometry, pole orientation, and shape of the asteroid. For details, the reader is refered to Eqs. 11 and 12 in Muinonen et al. (2015).

Using the relationship

$$
\frac{1}{8} \varpi_{0} P(\alpha)=p \frac{\phi_{H G_{1} G_{2}}(\alpha)}{\phi_{L S}(\alpha)},
$$

Eq. 5 can be re-written as

$$
\begin{aligned}
L\left(\alpha, e_{\odot}, e_{\oplus}\right)= & \pi F_{0} a b c p \frac{\phi_{H G_{1} G_{2}}(\alpha)}{\phi_{L S}(\alpha)} \frac{S_{\odot} S_{\oplus}}{S}\left\{\cos \left(\lambda^{\prime}-\alpha^{\prime}\right)+\cos \lambda^{\prime}+\sin \lambda^{\prime} \sin \left(\lambda^{\prime}-\alpha^{\prime}\right)\right. \\
& \left.\times \ln \left[\cot \frac{1}{2} \lambda^{\prime} \cot \frac{1}{2}\left(\alpha^{\prime}-\lambda^{\prime}\right)\right]\right\}
\end{aligned}
$$

where

$$
\phi_{L S}(\alpha)=1-\sin \frac{1}{2} \alpha \tan \frac{1}{2} \alpha \ln \left(\cot \frac{1}{4} \alpha\right) .
$$

$\phi_{L S}(\alpha)$ is the Lommel-Seeliger disk-integrated phase function for a spherical asteroid and $p$ is the geometric albedo. In the model above, the $H, G_{1}, G_{2}$ phase function (Muinonen et al. (2010) ) is incorporated into the LS-ellipsoid brightness model.

In short, altogether 12 unknown parameters are involved in the LSellipsoid model. They are the rotational period $\operatorname{Per}$, pole orientation $(\lambda, \beta)$ in the ecliptic frame of J2000.0, rotational phase $\varphi_{0}$ at $J D_{0}$, three semimajor axes $(a, b, c)$, geometric albedo $p$, phase function parameters $H, G_{1}, G_{2}$, and the equivalent diameter of asteroid $D$. To derive the solution of those unknown parameters, the flexible Nelder-Mead downhill method and a Markovchain Monte Carlo method (MCMC) are applied in our photometric analysis 
procedure. In practice, the analysis procedure of 2005 UD consists of two parts: the shape inversion of 2005 UD with the LS-ellipsoid model and the phase curve inversion. In the first part, we estimate rotation period, pole longitude and latitude, and three semimajor axes using 14 dense lightcurves. In the second part, using calibrated photometric data of $2005 \mathrm{UD}$, the phase curve parameters $H, G_{1}, G_{2}$ are retrieved.

\subsection{Shape inversion}

Altogether 14 dense lightcurves of 2005 UD are used to invert the spin and shape parameters by the Nelder-Mead downhill simplex method. Furthermore, the uncertainties of the parameters are assessed by the MCMC method.

Using the downhill simplex least-squares method, the following $\chi^{2}$ (Par) is minimized:

$$
\chi^{2}(\text { Par })=\sum_{i=1}^{N_{0}} \sum_{j=1}^{N_{i}} \frac{1}{\sigma_{i j}^{2}}\left[L_{o b s, i j}-L_{i j}(\text { Par })\right]^{2} .
$$

In practical shape inversion, only 7 parameters $\left(P e r, \lambda, \beta, \varphi_{0}, a, b, c\right)$ are estimated and the rest of the parameters are kept fixed in Eq. 7. Here $N_{0}$ is the number of lightcurves used, $N_{i}$ is the number of data points in the $i$ th lightcurve, $L_{o b s, i j}$ is the $j$ th data point in the $i$ th lightcurve, and $\sigma_{i j}$ is its corresponding uncertainty. $L_{i j}($ Par $)$ is the modeled brightness calculated with the LS-ellipsoid model.

To find the most probable rotation period, a wide range of periods within $2.5-12.5 \mathrm{~h}$ was scanned with a step of $\mathrm{Per}^{2} / 2 \mathrm{~T}$ (here $\mathrm{Per}$ is the assumed 
period and $T$ is the time span of all involved data). Fig. 3 shows the $\chi^{2}$ of the lightcurve fitting versus the trial period, the most likely value of period is located at $5.2338 \mathrm{~h}$. The result is close to Kinoshita's result of $5.2310 \mathrm{~h}$ (Kinoshita et al. (2007)). First, at each step of period scanning, hundreds of different initial poles distributed uniformly over the unit sphere are tested. Second, we have scanned the entire unit sphere with a step of $1^{\circ}$ in longitude and latitude directions to find the most probably pole of 2005 UD using the period of $5.2338 \mathrm{~h}$ as the initial value. The contours of $\chi^{2}$ versus the trial poles are shown in Fig. 4. The areas in blue color in Fig. 4 are corresponding to relatively small $\chi^{2}$. Two candidate poles of $\left(73^{\circ},-84^{\circ}\right)$ and $\left(285^{\circ},-21^{\circ}\right)$ are found with almost equal values of $\chi^{2}$. Third, taking the scanned spin parameters as initial values, unknown parameters are resolved with the Nelder-Mead downhill simplex method. Finally, we arrive at the following pair of pole solutions: Pole 1 at $\left(72^{\circ} .6^{,}-84^{\circ} .6\right)$ with axial ratios of $b / a=0.76, c / a=0.40$ and Pole 2 at $\left(285^{\circ} .8,-25^{\circ} .8\right)$ with axial ratios of $b / a=0.76$ and $c / a=0.40$. The periods corresponding to the poles are close to $5.2338 \mathrm{~h}$.

In order to derive the uncertainties for the spin and shape parameters of $2005 \mathrm{UD}$, an MCMC simulation is run based on the photometric data of 2005 UD and the LS-ellipsoid model. The a posteriori probability density for the parameters is characterized by a large number of sample solutions obtained by Metropolis-Hastings sampling. The proposal densities for the parameters are constructed via a collection of virtual least-squares solutions derived from virtual photometric data. The virtual photometric data are 


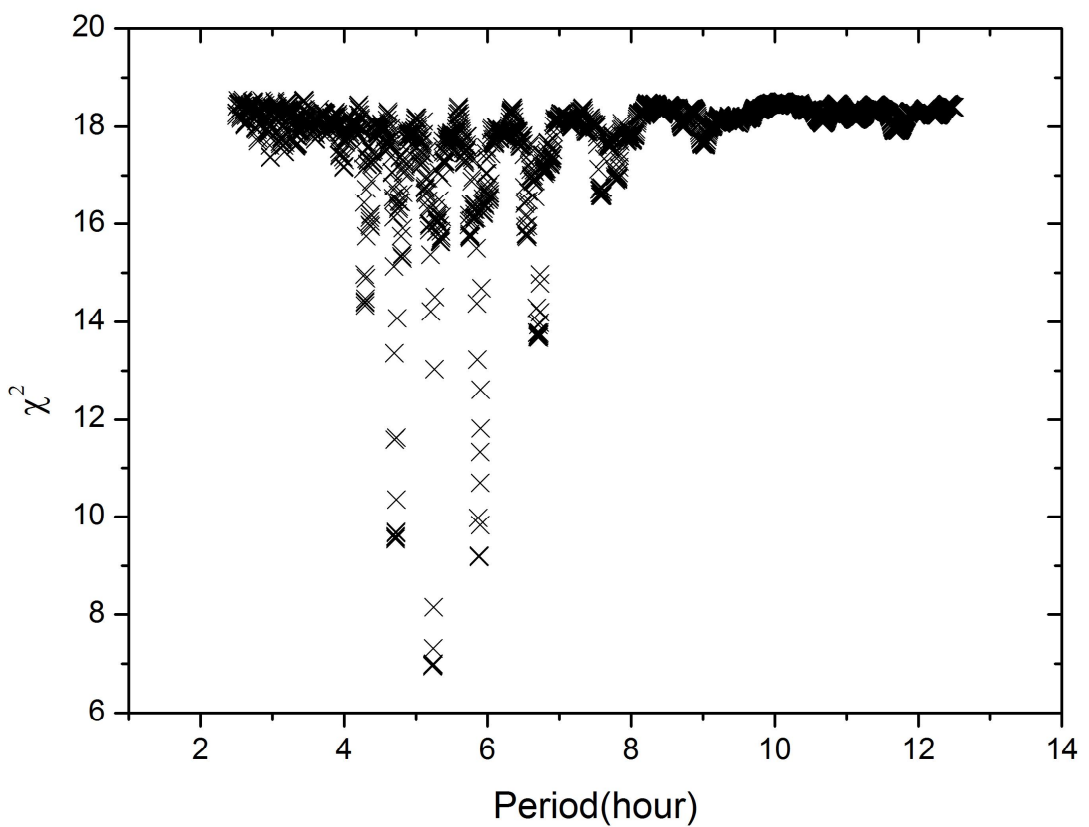

Figure 3: The $\chi^{2}$-values for the trial periods.

generated by adding Gaussian noise into the observations. At least 10000 samples are obtained with the MCMC simulation. The joint distributions of the spin parameters are shown in Fig. 5 for Pole 1 and Fig. 6 for Pole 2. The dotted lines in Figs. 5 and 6 are the best-fit values of the parameters. The intervals between the best-fit value and the $1-\sigma$ limits for each distribution are used to estimate the uncertainties of the parameters. Based on those joint distributions, the best-fit values of the parameters with their uncertainties are as follows: Pole 1 at $\left(72^{\circ} .6_{-7.3}^{+4.2},-84^{\circ} .6_{-2.1}^{+6.2}\right)$ with axial ratios $b / a=0.75_{-0.01}^{+0.01}$ and $c / a=0.40_{-0.01}^{+0.16}$ and Pole 2 at $\left(285^{\circ} .8_{-5.3}^{+1.1},-25^{\circ} .8_{-12.5}^{+5.3}\right)$ with axial ratios $b / a=0.76_{-0.01}^{+0.01}$ and $c / a=0.40_{-0.01}^{+0.03}$. The periods corresponding to the 

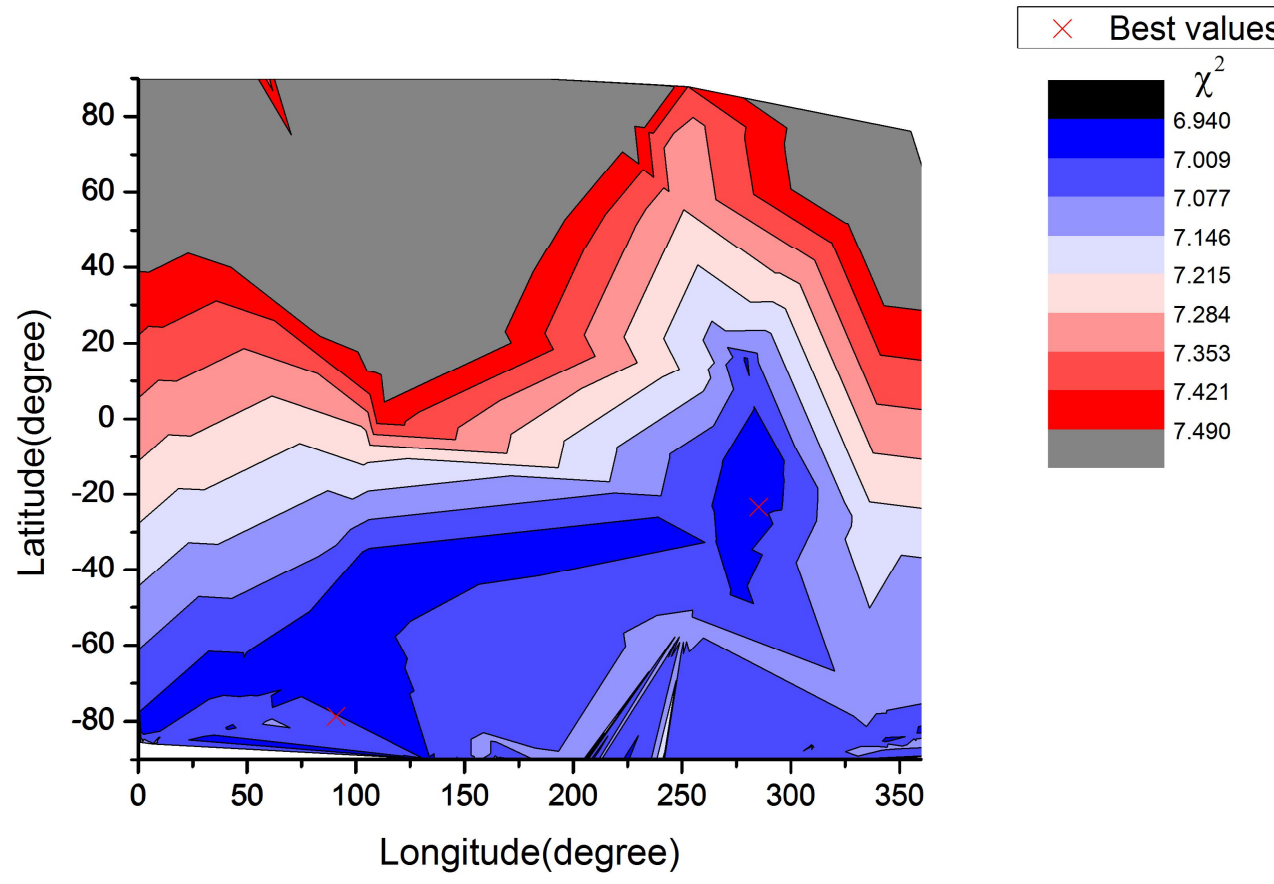

Figure 4: The $\chi^{2}$-values for the trial pole orientations.

poles are $5.23351_{-0.00001}^{+0.00002}$ and $5.23403_{-0.00001}^{+0.00004}$, respectively. Comparing the distributions of the poles, the solution of Pole 2 is prefered. Indeed, which one of the pole solutions is the true solution requires more observations, even observations with other techniques (e.g., imaging, occultation, or radar).

In order to understand the inversion results intuitively, the model brightnesses from the solution with Pole 2 solution are shown in Fig. 1 together with the observations. Most observed brightnesses are fitted well by the modeled brightness, but some data (e.g., data obtained on on 10 Oct. 2018) are not. This minor caveat may be due to the fast sky-plane motion of the asteroid, resulting in a low quality of photometric data due to the elongated 
image of the asteroid.
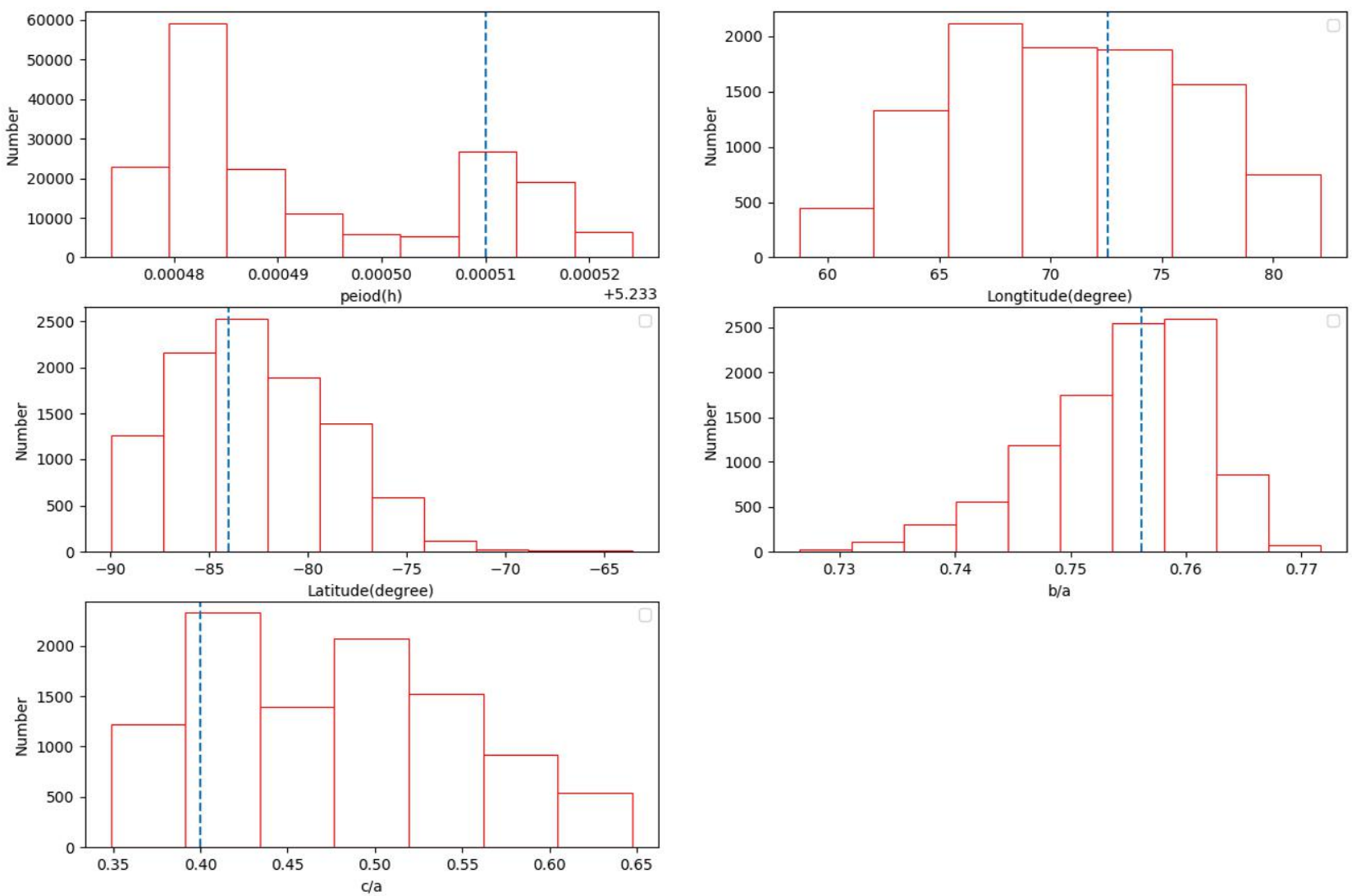

Figure 5: Joint distributions of spin and shape parameters for Pole 1.

\subsection{Phase curve fitting}

The photometric phase curve of an asteroid shows the observed brightness variation as a function of the solar phase angle. There are two phase 
functions, the $H, G$ and $H, G_{1}, G_{2}$ phase functions, to be used to describe the photometric phase curves of asteroids. The $H, G$ system ( $H$ and $G$ being the absolute magnitude and slope parameter of the asteroid, respectively) has been adopted by the International Astronomical Union (IAU) in 1985 as the standard photometric system for asteroids, developed from the Lumme-Bowell model (Bowell et al. (1989)). The slope parameter $G$ characterizes an asteroid's surface, whereas the absolute magnitude $H$ is related to its size and geometric albedo. The $H, G_{1}, G_{2}$ phase function is used in the standard photometric system of asteroids adopted by the IAU in 2012 . The new three-parameter phase function improves the fits of phase curves of both high-albedo and low-albedo asteroids. Here, we use the $H, G_{1}, G_{2}$ phase function to fit the calibrated photometric data of 2005 UD. The basic model for linear least-squares fitting is

$$
\begin{aligned}
10^{-0.4 V(\alpha)} & =a_{1} \phi_{1}(\alpha)+a_{2} \phi_{2}(\alpha)+a_{3} \phi_{3}(\alpha) \\
& =10^{-0.4 H}\left[G_{1} \phi_{1}(\alpha)+G_{2} \phi_{2}(\alpha)+\left(1-G_{1}-G_{2}\right) \phi_{3}(\alpha)\right) .
\end{aligned}
$$

The source code for $H, G_{1}, G_{2}$ phase curve inversion is openly available 8 . In practice, the phase curve inversion is carried out based on the disk-integrated brightnesses of the asteroid (see Eq. 8). The linear parameters $a_{1}, a_{2}$, and $a_{3}$ are derived first, then the parameters $H, G_{1}$, and $G_{2}$ are calculated using Eq. 19 in Muinonen et al. (2010). The functions $\phi_{1,2,3}(\alpha)$ are basis functions expressed in cubic splines with the interpolation grid of phase angles of

\footnotetext{
$\S$ http://h152.it.helsinki.fi/HG1G2/
} 
$\left(0^{\circ}, 0.3^{\circ}, 1^{\circ}, 2^{\circ}, 4^{\circ}, 8^{\circ}, 15^{\circ}, 30^{\circ}, 60^{\circ}, 90^{\circ}, 120^{\circ}\right.$, and $\left.150^{\circ}\right)$. The detailed values are available in Tables 3 and 4 in Muinonen et al. (2010)).

The mean values of the dense lightcurves and ZTF data are used to fit the $H, G_{1}, G_{2}$ function with the MCMC method. In the MCMC simulation procedure, the Metropolis-Hastings algorithm is applied to sample using the Gaussian proposal probability densities of the parameters. Fig. 7 shows the joint distributions of the three parameters. The best values of $H, G_{1}$, and $G_{2}$ are $17.19 \mathrm{mag}, 0.573$, and 0.004 . Using $1-\sigma$ limits of the distributions, we obtain $H=17.19_{-0.09}^{+0.10} \mathrm{mag}, G_{1}=0.573_{-0.069}^{+0.088}, G_{2}=0.004_{-0.021}^{+0.020}$. The bestfit model to the photometric data is displayed in Fig. 2. From the best-fit values of $G_{1}$ and $G_{2}, 2005 \mathrm{UD}$ is likely to be a C-type asteroid according to the suggestion in Shevchenko et al. (2016).

\section{Summary}

In order to study the near-Earth asteroid 2005 UD, we carried out 11 nights of photometric observations with a $30-\mathrm{cm}$ telescope at the Corona borealis Observatory in Ali, Tibet, China. Combining our 11 lightcurves with the 3 lightcurves from the MPC ALCDEP database, the spin and shape parameters of 2005 UD have been analysed with the Lommel-Seeliger ellipsoid method. Two pole solutions, Pole 1 at $\left(72^{\circ} .6_{-7.3}^{+4.2},-84^{\circ} .6_{-2.1}^{+6.2}\right)$ and Pole 2 at $\left(285^{\circ} .8_{-5.3}^{+1.1},-25^{\circ} .8_{-12.5}^{+5.3}\right)$, have been derived. Comparing the distributions of spin parameters, we prefer Pole 2, since it gives more concentrated distributions. The axial ratios of the ellipsoid corresponding to Pole 2 are $b / a=0.76_{-0.01}^{+0.01}, c / a=0.40_{-0.01}^{+0.03}$. The spin period is $5.23403_{-0.00001}^{+0.00004} \mathrm{~h}$. 
The distribution of the pole latitude (see Figs. 5 and 6) is wider than that of the pole longitude. This is due to a small span of the aspect angles of the photometric data. So more photometric observations are necessary for improving the pole orientation, especial in latitude.

Our group also focuses on the physical studies of another PGC member, that is, asteroid (3200) Phaethon. We have found that Pole 2 of 2005 UD is close to that of Phaethon. Investigating published pole solutions of Phaethon, we have noted that they show slight differences: for example, $\left(97^{\circ} \pm 10^{\circ},-11^{\circ} \pm\right.$ $\left.10^{\circ}\right)$ and $\left(276^{\circ} \pm 10^{\circ},-15^{\circ} \pm 10^{\circ}\right)$ from Krugly et al. (2002), $\left(85^{\circ},-20^{\circ}\right)$ from Ansdell et al. (2014), $\left(319^{\circ},-39^{\circ}\right)$ from Hanuš et al. (2016), and $\left(308^{\circ} \pm\right.$ $\left.10^{\circ},-52^{\circ} \pm 10^{\circ}\right)$ and $\left(322^{\circ} \pm 10^{\circ},-40^{\circ} \pm 10^{\circ}\right)$ fromKim et al. (2018). Our group gives a pair of poles of $\left.\left(95^{\circ} .9,-20^{\circ} .4\right)\right)$ and $\left(311^{\circ} .2,-23^{\circ} .6\right)$ (a paper is being prepared). If considering the second pole solution of Phaethon, above pole solutions are around three directions: $(a)\left(276^{\circ} \pm 10^{\circ},-15^{\circ} \pm 10^{\circ}\right)$, (b) $\left(311^{\circ} .2,-23^{\circ} .6\right)$ and $(c)\left(319^{\circ},-39^{\circ}\right)$. The differences among the three orientations occur in ecliptic latitude: $-15^{\circ},-23^{\circ}$, and $-39^{\circ}$. In the case of (b) and (c), the longitudes are very close to each other, whereas in the case of (a), the longitude diverges from that of (b) and (c). Considering the uncertainty of the pole solutions, Phaethon's pole appears to align with that of 2005 UD. If it is true, we think this evidence implies 2005 UD probably originated via a collision or rotational fission from Phaethon's parent body, resembling the case of the Koronis family (Slivan (2002)).

Combining the mean magnitudes of the dense lightcurves and the ZTF data, we have fitted the photometric phase curve of 2005 UD with the three 
parameter $H, G_{1}, G_{2}$ phase function. The parameters $H, G_{1}$ and $G_{2}$ are as follows: $17.19_{-0.09}^{+0.10} \mathrm{mag}, 0.573_{-0.069}^{+0.088}, 0.004_{-0.021}^{+0.020}$, respectively. Given these values of the parameters, the phase integral parameter $q$, normalized slope of the phase-curve $k$ (within $\alpha\left(7^{\circ} .5\right)$ and the amplitude of the opposition effect $\zeta-1$ ( $\zeta$ is the enhancement factor) are estimated to be $0.2441,-1.9076$, and 0.7309 as computed from the following relationships(Muinonen et al. (2010)):

$$
\begin{gathered}
q=0.009082+0.4061 G_{1}+0.8092 G_{2}, \\
k=-\frac{G_{1} \frac{6}{\pi}+G_{2} \frac{9}{5 \pi}}{G_{1}+G_{2}}=-\frac{1}{5 \pi} \frac{30 G_{1}+9 G_{2}}{G_{1}+G_{2}}, \\
\zeta-1=\frac{1-G_{1}-G_{2}}{G_{1}+G_{2}} .
\end{gathered}
$$

Based on the derived $H$ value and the relationship of the diameter and albedo $\left(D=\frac{1329}{\sqrt{p_{v}}} 10^{-0.2 H}\right)$ (Bowell et al. 1989), the equivalent diameter $D$ of 2005 UD is estimated to be $1.3 \mathrm{~km}$ using its new derived albedo of $0.14 \pm 0.09$ (Masiero et al. (2019)), which is slightly higher than the previously estimated value of $1.2 \pm 0.4 \mathrm{~km}$ (Masiero et al. (2019)).

\section{Acknowledgements}

The research has been funded by the National Natural Science Foundation of China (Grant Nos. 11073051 and 11673063) and the Academy of Finland (Grant No. 325805). The research has made use of the NASA/IPAC Infrared Science Archive, which is funded by the National Aeronautics and Space Administration and operated by the California Institute of Technology. The work includes data from the Asteroid Terrestrial-impact Last Alert 
System (ATLAS) project. ATLAS is primarily funded to search for nearEarth asteroids through NASA grants NN12AR55G, 80NSSC18K0284, and 80NSSC18K1575; byproducts of the near-Earth-object search include images and catalogs from the survey area. The ATLAS science products have been made possible through the contributions of the University of Hawaii Institute for Astronomy, the Queen's University Belfast, the Space Telescope Science Institute, and the South African Astronomical Observatory.

\section{References}

Ansdell, M., Meech, K.J., Hainaut, O., Buie, M.W., Kaluna, H., Bauer, J., Dundon, L., 2014. Refined Rotational Period, Pole Solution, and Shape Model for (3200) Phaethon. The Astrophysical Journal 793, 50. doi:10. 1088/0004-637X/793/1/50, arXiv:1407.7886.

Bellm, E.C., Kulkarni, S.R., Graham, M.J., Dekany, R., Smith, R.M., Riddle, R., Masci, F.J., Helou, G., Prince, T.A., Adams, S.M., Barbarino, C., Barlow, T., Bauer, J., Beck, R., Belicki, J., Biswas, R., Blagorodnova, N., Bodewits, D., Bolin, B., Brinnel, V., Brooke, T., Bue, B., Bulla, M., Burruss, R., Cenko, S.B., Chang, C.K., Connolly, A., Coughlin, M., Cromer, J., Cunningham, V., De, K., Delacroix, A., Desai, V., Duev, D.A., Eadie, G., Farnham, T.L., Feeney, M., Feindt, U., Flynn, D., Franckowiak, A., Frederick, S., Fremling, C., Gal-Yam, A., Gezari, S., Giomi, M., Goldstein, D.A., Golkhou, V.Z., Goobar, A., Groom, S., Hacopians, E., Hale, D., Henning, J., Ho, A.Y.Q., Hover, D., Howell, J., Hung, T., Huppenkothen, D., 
Imel, D., Ip, W.H., Ivezić, Ž., Jackson, E., Jones, L., Juric, M., Kasliwal, M.M., Kaspi, S., Kaye, S., Kelley, M.S.P., Kowalski, M., Kramer, E., Kupfer, T., Landry, W., Laher, R.R., Lee, C.D., Lin, H.W., Lin, Z.Y., Lunnan, R., Giomi, M., Mahabal, A., Mao, P., Miller, A.A., Monkewitz, S., Murphy, P., Ngeow, C.C., Nordin, J., Nugent, P., Ofek, E., Patterson, M.T., Penprase, B., Porter, M., Rauch, L., Rebbapragada, U., Reiley, D., Rigault, M., Rodriguez, H., van Roestel, J., Rusholme, B., van Santen, J., Schulze, S., Shupe, D.L., Singer, L.P., Soumagnac, M.T., Stein, R., Surace, J., Sollerman, J., Szkody, P., Taddia, F., Terek, S., Van Sistine, A., van Velzen, S., Vestrand, W.T., Walters, R., Ward, C., Ye, Q.Z., Yu, P.C., Yan, L., Zolkower, J., 2019. The Zwicky Transient Facility: System Overview, Performance, and First Results. Publications of the Astronomical Society of the Pacific 131, 018002. doi:10.1088/1538-3873/aaecbe, arXiv:1902.01932.

Bowell, E., Hapke, B., Domingue, D., Lumme, K., Peltoniemi, J., Harris, A.W., 1989. Application of photometric models to asteroids., in: Binzel, R.P., Gehrels, T., Matthews, M.S. (Eds.), Asteroids II, pp. 524-556.

Bowell, E., Lumme, K., 1979. Colorimetry and magnitudes of asteroids.

Collier Cameron, A., Pollacco, D., Street, R.A., Lister, T.A., West, R.G., Wilson, D.M., Pont, F., Christian, D.J., Clarkson, W.I., Enoch, B., Evans, A., Fitzsimmons, A., Haswell, C.A., Hellier, C., Hodgkin, S.T., Horne, K., Irwin, J., Kane, S.R., Keenan, F.P., Norton, A.J., Parley, N.R., Osborne, J., Ryans, R., Skillen, I., Wheatley, P.J., 2006. A fast hybrid algorithm 
for exoplanetary transit searches. Monthly Notices of the Royal Astronomical Society 373, 799-810. doi:10.1111/j.1365-2966.2006.11074.x, arXiv:astro-ph/0609418.

Dymock, R., Miles, R., 2009. A method for determining the V magnitude of asteroids from CCD images. Journal of the British Astronomical Association 119, 149-156. arXiv:1006.4017.

Hanuš, J., Ďurech, J., Oszkiewicz, D.A., Behrend, R., Carry, B., Delbo, M., Adam, O., Afonina, V., Anquetin, R., Antonini, P., Arnold, L., Audejean, M., Aurard, P., Bachschmidt, M., Baduel, B., Barbotin, E., Barroy, P., Baudouin, P., Berard, L., Berger, N., Bernasconi, L., Bosch, J.G., Bouley, S., Bozhinova, I., Brinsfield, J., Brunetto, L., Canaud, G., Caron, J., Carrier, F., Casalnuovo, G., Casulli, S., Cerda, M., Chalamet, L., Charbonnel, S., Chinaglia, B., Cikota, A., Colas, F., Coliac, J.F., Collet, A., Coloma, J., Conjat, M., Conseil, E., Costa, R., Crippa, R., Cristofanelli, M., Damerdji, Y., Debackère, A., Decock, A., Déhais, Q., Déléage, T., Delmelle, S., Demeautis, C., Dróżdż, M., Dubos, G., Dulcamara, T., Dumont, M., Durkee, R., Dymock, R., Escalante del Valle, A., Esseiva, N., Esseiva, R., Esteban, M., Fauchez, T., Fauerbach, M., Fauvaud, M., Fauvaud, S., Forné, E., Fournel, C., Fradet, D., Garlitz, J., Gerteis, O., Gillier, C., Gillon, M., Giraud, R., Godard, J.P., Goncalves, R., Hamanowa, H., Hamanowa, H., Hay, K., Hellmich, S., Heterier, S., Higgins, D., Hirsch, R., Hodosan, G., Hren, M., Hygate, A., Innocent, N., Jacquinot, H., Jawahar, S., Jehin, E., Jerosimic, L., Klotz, A., Koff, W., Korlevic, P., Kosturkiewicz, E., Krafft, 
P., Krugly, Y., Kugel, F., Labrevoir, O., Lecacheux, J., Lehký, M., Leroy, A., Lesquerbault, B., Lopez-Gonzales, M.J., Lutz, M., Mallecot, B., Manfroid, J., Manzini, F., Marciniak, A., Martin, A., Modave, B., Montaigut, R., Montier, J., Morelle, E., Morton, B., Mottola, S., Naves, R., Nomen, J., Oey, J., Ogłoza, W., Paiella, M., Pallares, H., Peyrot, A., Pilcher, F., Pirenne, J.F., Piron, P., Polińska, M., Polotto, M., Poncy, R., Previt, J.P., Reignier, F., Renauld, D., Ricci, D., Richard, F., Rinner, C., Risoldi, V., Robilliard, D., Romeuf, D., Rousseau, G., Roy, R., Ruthroff, J., Salom, P.A., Salvador, L., Sanchez, S., Santana-Ros, T., Scholz, A., Séné, G., Skiff, B., Sobkowiak, K., Sogorb, P., Soldán, F., Spiridakis, A., Splanska, E., Sposetti, S., Starkey, D., Stephens, R., Stiepen, A., Stoss, R., Strajnic, J., Teng, J.P., Tumolo, G., Vagnozzi, A., Vanoutryve, B., Vugnon, J.M., Warner, B.D., Waucomont, M., Wertz, O., Winiarski, M., Wolf, M., 2016. New and updated convex shape models of asteroids based on optical data from a large collaboration network. Astronomy and Astrophysics 586, A108. doi:10.1051/0004-6361/201527441, arXiv: 1510.07422.

Hapke, B., 2012. Bidirectional reflectance spectroscopy 7. The single particle phase function hockey stick relation. Icarus 221, 1079-1083. doi:10.1016/ j.icarus.2012.10.022.

Jewitt, D., 2013. Properties of Near-Sun Asteroids. The Astronomical Journal 145, 133. doi:10.1088/0004-6256/145/5/133, arXiv:1303.2415.

Jewitt, D., Hsieh, H., 2006. Physical Observations of 2005 UD: A MiniPhaethon. The Astronomical Journal 132, 1624-1629. doi:10.1086/ 
Kasuga, T., Jewitt, D., 2008. Observations of 1999 YC and the Breakup of the Geminid Stream Parent. The Astronomical Journal 136, 881-889. doi:10.1088/0004-6256/136/2/881, arXiv:0805.2636.

Kim, M.J., Lee, H.J., Lee, S.M., Kim, D.H., Yoshida, F., Bartczak, P., Dudziński, G., Park, J., Choi, Y.J., Moon, H.K., Yim, H.S., Choi, J., Choi, E.J., Yoon, J.N., Serebryanskiy, A., Krugov, M., Reva, I., Ergashev, K.E., Burkhonov, O., Ehgamberdiev, S.A., Turayev, Y., Lin, Z.Y., Arai, T., Ohtsuka, K., Ito, T., Urakawa, S., Ishiguro, M., 2018. Optical observations of NEA 3200 Phaethon (1983 TB) during the 2017 apparition. Astronomy and Astrophysics 619, A123. doi:10.1051/0004-6361/201833593, arXiv:1809.05900,

Kinoshita, D., Ohtsuka, K., Sekiguchi, T., Watanabe, J., Ito, T., Arakida, H., Kasuga, T., Miyasaka, S., Nakamura, R., Lin, H.C., 2007. Surface heterogeneity of 2005 UD from photometric observations. Astronomy and Astrophysics 466, 1153-1158. doi:10.1051/0004-6361:20066276.

Krugly, Y., Belskaya, I.N., Mykhailova, S.S., Donchev, Z., Inasaridze, R.Y., Sergeyev, A.V., Slyusarev, I.G., Shevchenko, V.G., Chiorny, V.G., Rumyantsev, V.V., Novichonok, A.O., Ayvazian, V., Kapanadze, G., Kvaratskhelia, O.I., Bonev, T., Borisov, G., Molotov, I.E., Voropaev, V.A., 2019. Photometry and polarimetry of near-Earth asteroids (3200) 
Phaethon and (155140) 2005 UD, in: EPSC-DPS Joint Meeting 2019, pp. EPSC-DPS2019-1989.

Krugly, Y.N., Belskaya, I.N., Shevchenko, V.G., Chiorny, V.G., Velichko, F.P., Mottola, S., Erikson, A., Hahn, G., Nathues, A., Neukum, G., Gaftonyuk, N.M., Dotto, E., 2002. The Near-Earth Objects Followup Program. IV. CCD Photometry in 1996-1999. Icarus 158, 294-304. doi:10.1006/icar.2002.6884.

Laher, R.R., Surace, J., Grillmair, C.J., Ofek, E.O., Levitan, D., Sesar, B., van Eyken, J.C., Law, N.M., Helou, G., Hamam, N., Masci, F.J., Mattingly, S., Jackson, E., Hacopeans, E., Mi, W., Groom, S., Teplitz, H., Desai, V., Hale, D., Smith, R., Walters, R., Quimby, R., Kasliwal, M., Horesh, A., Bellm, E., Barlow, T., Waszczak, A., Prince, T.A., Kulkarni, S.R., 2014. IPAC Image Processing and Data Archiving for the Palomar Transient Factory. Publications of the Astronomical Society of the Pacific 126, 674. doi:10.1086/677351, arXiv:1404.1953.

Masiero, J.R., Wright, E.L., Mainzer, A.K., 2019. Thermophysical Modeling of NEOWISE Observations of DESTINY+ Targets Phaethon and 2005 UD. The Astronomical Journal 158, 97. doi:10.3847/1538-3881/ab31a6, arXiv:1907.04518.

Muinonen, K., Belskaya, I.N., Cellino, A., Delbò, M., Levasseur-Regourd, A.C., Penttilä, A., Tedesco, E.F., 2010. A three-parameter magnitude 
phase function for asteroids. Icarus 209, 542-555. doi:10.1016/j . icarus. 2010.04 .003

Muinonen, K., Wilkman, O., Cellino, A., Wang, X., Wang, Y., 2015. Asteroid lightcurve inversion with Lommel-Seeliger ellipsoids. Planetary and Space Science 118, 227-241. doi:10.1016/j.pss.2015.09.005.

Ofek, E.O., Laher, R., Law, N., Surace, J., Levitan, D., Sesar, B., Horesh, A., Poznanski, D., van Eyken, J.C., Kulkarni, S.R., Nugent, P., Zolkower, J., Walters, R., Sullivan, M., Agüeros, M., Bildsten, L., Bloom, J., Cenko, S.B., Gal-Yam, A., Grillmair, C., Helou, G., Kasliwal, M.M., Quimby, R., 2012. The Palomar Transient Factory Photometric Calibration. Publications of the Astronomical Society of the Pacific 124, 62. doi:10.1086/664065, arXiv:1112.4851.

Ohtsuka, K., Sekiguchi, T., Kinoshita, D., Watanabe, J.I., Ito, T., Arakida, H., Kasuga, T., 2006. Apollo asteroid 2005 UD: split nucleus of (3200) Phaethon? Astronomy and Astrophysics 450, L25-L28. doi:10.1051/ 0004-6361:200600022.

Shevchenko, V.G., Belskaya, I.N., Muinonen, K., Penttilä, A., Krugly, Y.N., Velichko, F.P., Chiorny, V.G., Slyusarev, I.G., Gaftonyuk, N.M., Tereschenko, I.A., 2016. Asteroid observations at low phase angles. IV. Average parameters for the new $\mathrm{H}, \mathrm{G}_{1}, \mathrm{G}_{2}$ magnitude system. Planetary and Space Science 123, 101-116. doi:10.1016/j.pss.2015.11.007. 
Slivan, S.M., 2002. Spin vector alignment of Koronis family asteroids. Nature 419, 49-51. doi:10.1038/nature00993.

Tamuz, O., Mazeh, T., Zucker, S., 2005. Correcting systematic effects in a large set of photometric light curves. Monthly Notices of the Royal Astronomical Society 356, 1466-1470. doi:10.1111/j.1365-2966.2004. 08585.x, arXiv:astro-ph/0502056.

Wang, X.B., Gu, S.H., Collier Cameron, A., Tan, H.B., Hui, H.K., Kwok, C.T., Yeung, B., Leung, K.C., 2013. The refined physical parameters of transiting exoplanet system HAT-P-24. Research in Astronomy and Astrophysics 13, 593-603. doi:10.1088/1674-4527/13/5/010.

Warner, B.D., Stephens, R.D., 2019. Near-Earth Asteroid Lightcurve Analysis at the Center for Solar System Studies: 2018 September-December. Minor Planet Bulletin 46, 144-152. 



Figure 6: Joint distributions of spin and shape parameters for Pole 2. 



Figure 7: Marginal distributions of $H, G_{1}, G_{2}$ for 2005 UD. 